\title{
Optimizing power generation in the presence of micro-grids
}

\author{
Wim van Ackooij ${ }^{\mathrm{a}}$, Jérôme De Boeck ${ }^{\mathrm{b}}$, Boris Detienne ${ }^{\mathrm{c}}$, Stefania Pan ${ }^{\mathrm{d}}$, Michael Poss $^{\mathrm{e}}$ \\ ${ }^{a}$ EDF R\&D. OSIRIS, 7, Boulevard Gaspard Monge, F-91120 Palaiseau Cedex France. \\ ${ }^{b}$ Department of Computer Science, Université Libre de Bruxelles, B-1050 Brussels, Belgium and INOCS, INRIA Lille \\ Nord-Europe, France \\ ${ }^{c}$ Institute of Mathematics, University of Bordeaux, Inria Bordeaux-Sud-Ouest, 153 cours de la libération, 33400 \\ Talence, France \\ ${ }^{d}$ UMR 7030 CNRS LIPN, Université Paris 13, 99 avenue Jean-Baptiste Clément, 93430 Villetaneuse, France \\ ${ }^{e}$ UMR CNRS 5506 LIRMM, Université de Montpellier, 161 rue Ada, 34392 Montpellier Cedex 5, France.
}

\begin{abstract}
In this paper we consider energy management optimization problems in a future wherein an interaction with micro-grids has to be accounted for. We will model this interaction through a set of contracts between the generation companies owning centralized assets and the micro-grids. We will formulate a general stylized model that can, in principle, account for a variety of management questions such as unit-commitment. The resulting model, a bilevel stochastic mixed integer program will be numerically tackled through a novel preprocessing procedure. As a result the solution for the bilevel (or single leader multiple follower) problem will be neither "optimistic" nor "pessimistic". We will numerically evaluate the difference of the resulting solution with the "optimistic" solution. We will also demonstrate the efficiency and potential of our methodology on a set of numerical instances.
\end{abstract}

Keywords: integer programming, power generation, bilevel optimization, stochastic optimization, single leader multiple follower games

2010 MSC: 90C10, 90C27

\section{Introduction}

In the upcoming future the energy landscape will significantly change from the current picture by incorporating more and more decentralized elements. Of particular importance is the advent of so-called micro-grids (MG). These are subparts of the system with an advanced energy management system interacting with programmable elements in the grid including good monitoring and control functions, a pervasive communication system and specific items such as smart meters, programmable loads, switchable storage systems and a variety of controllable energy sources including solar, wind and wave generators. Some of the major changes introduced by smart grids are the following:

- Micro-grids: smaller nearly isolated sub-grids that interact only with the global system when a

Email addresses: wim.van-ackooij@edf.fr (Wim van Ackooij), jdeboeck@ulb.ac.be (Jérôme De Boeck), michael.poss@lirmm.fr (Michael Poss)

Preprint submitted to European Journal of Operational Research

May 18, 2018 
load/offer mismatch occurs. Most importantly these sub-grids can be managed to follow a local economical target (which may be different and contrary to a system-wide interest).

- Partial storage, perhaps through electrical vehicles or powerful batteries. These storage devices can partially mitigate the intermittency of local decentralized production such as wind / solar generation.

- Demand management tools: use advanced information technology to pilot electricity use. For instance, shut down electrical heating, reprogram hot water tank recharging etc...

In this new setting it becomes of great interest to examine the interaction with more traditional elements composing the power system. For instance what will be the new role for large centralized generation assets such as nuclear, thermal or hydro generation? It also becomes of interest to examine how classical energy management questions, such as unit-commitment (e.g., [1,2]), scheduling maintenance of large power plants or cascaded reservoir management (e.g., $[3,4]$ ) should evolve to account for this new context. Needless to say, these classical management questions are already difficult on their own without considering a potential interaction with micro-grids. On top of this comes also the need to consider and account for uncertainty which are of great importance for obtaining meaningful management solutions.

The interaction between such micro-grids and centralized assets may be mathematically cast into the setting of a "game". We will make the specific choice of considering hierarchical games wherein a (single) large centralized operator (the leader or upper level) interacts with one or several micro-grids (the follower(s) or lower level) in a specific way. Such problems also go by the name of single leader multifollower or in the case of a single follower by the naming of Stackelberg game or principal agent problems. We follow the terminology of [5] speaking of bilevel optimization problems, i.e., an optimization problem containing constraints that certain variables belong to the optimal set of other parametric optimization problems. Generally speaking these problems fall into the class of mathematical problems with equilibrium constraints, where $[6,7]$ are key references. The situation with multiple leaders is significantly harder to analyse and falls into the class of Equilibrium problems with Equilibrium constraints (EPECs). We refer to, e.g., [8-11] for some applications in energy and to e.g., [12, 13] and references therein for structural studies. Under some appropriate structure, a bilevel optimization problem involving only continuous variables can be cast as a DC (difference of convex) - problem. We refer to [14, 15] for more on such methodology.

In [16] the authors claim to investigate bilevel programming in the electric utility industry for the first time. The lower level (or follower), representing customers deals with maximizing benefit while investing in energy conservation. From a technical viewpoint, the authors substitute the Karush-Kuhn-Tucker (KKT) conditions for the lower level problem and process the subsequent mathematical program with equilibrium constraints (MPEC), by employing an exhaustive search on the complementarity conditions (one classic problem is solved for each state of the complementarity conditions). This procedure means that for an $n$ dimensional complementarity constraint, $2^{n}$ problems need to be solved. Substituting the lower level KKT conditions in order to obtain a one-level more classic problem is commonplace in the energy literature (e.g., [17-19] just to name a few). However it should be noted that, following [20], this does not necessarily lead to an equivalent formulation of the original problem. In other words, the solution resulting from the KKT reformulation need not be a solution to the original bilevel program. This phenomenon can occur even in the simplest linear setting. We also refer to the recent [21] illustrating a similar phenomenon. The essential observation is that the original problem is augmented with a given set of Lagrange multipliers. Consequently any local solution to the original problem must be a local solution to the augmented problem for all such Lagrange multipliers. However, whenever the augmented 
problem is solved, one ends up with just one specific Lagrange multiplier. In principle it should thus be verified if the thus obtained solution remains a local solution for all other Lagrange multipliers. Yet these are in general not readily available. For a simple 2 variable example of a situation wherein the KKT reformulation provides local solutions that are not local solutions of the original problem, see [5, Example 3.1]. The authors of [22] also consider a leader-follower framework in the electrical industry but rather in the objective of benchmarking a given investment strategy in cogeneration against a centrally planned strategy. In order to do so, they develop an iterative algorithm, which numerically seems to converge to a Betrand-Cournot equilibrium. The comparison with the centrally planned solution reveals inefficiencies of the given investment strategy.

Recently, demand side management (or demand response) was recognized to be an important aspect of the upcoming electrical system. As such, the authors of [23] claim to be the first to consider such demand response within a bilevel framework. The lower level (or follower) problem is an optimal power flow problem under a direct current assumption. The lower level power balance equation and its dual multiplier in turn help fixing the global price of energy in the upper level (or leader) problem (e.g., $[24,25]$ suggest a similar model but without solving it). From a methodological viewpoint, bilinear terms are linearized using McCormick envelopes, the lower level KKT conditions are substituted for the lower level problem and the special model structure can be exploited through several substitutions. In view of those claims our work is one of the first to consider the effect of demand side management on classical energy management questions. Moreover we will highlight that demand side management is responsible for ensuring that the optimistic and pessimistic solutions of the bilevel program are not identical. Although in our work we will focus on the interaction between a utility and several end-users grouped within one or several smart-grids, we will not consider the interaction between users as done in [26] where the focus is on reducing peak-to-average load ratios while accounting for potential games between users. As usual in these works, we too will adopt a market-like setting wherein a predetermined set of contracts is offered to the customers.

In this paper we thus contribute to this difficult question by considering a stylized interaction between a generation company (GenCo) owning a (large) set of centralized assets and a set of micro-grids. We also account for potential competition at the centralized level, but only in a simplified way. Indeed we will make the assumption that any competitors to the GenCo have a fixed predetermined interaction with the micro-grids. In this work the GenCo can offer contracts to the micro-grid that detail the price of buying/selling electricity to the network. From a mathematical viewpoint we suggest a bilevel stochastic mixed integer program (BMIP) which in principle can account for a variety of instantiations thus covering any of the above management questions. In our numerical experiments however, we will focus on unit-commitment.

The first general methods for obtaining "optimistic" optimal solutions BMIPs can be traced back to [27] and [28], which could solve only small instances, with up to 10 general integer variables and 35 binary variables for the upper level problem. While more recent works have shown significant improvements (e.g., [29] based on intersection cuts), they are unable to handle the complex MILPs involved in unitcommitments or more complex power flow optimization problems. Our technical contribution therefore lies in suggesting a tractable reformulation of the bilevel problem that leads to a solution that is neither "optimistic" nor "pessimistic". This "heuristic" reformulation is tractable thanks to a preprocessing step that is not harder than solving the original management problem. We also compare the solution obtained from this reformulation with the "optimistic" solution that can only be obtained in reasonable times for small systems. Extensive numerical experiments confirm the interest of the here suggested reformulation. Essentially our reformulation takes inspiration, and to some degree is equivalent with, the value function approach originally designed by Outrata [30]. An advantage of this approach over the 
usual substitution of the KKT conditions is that we do obtain a solution of the original problem, albeit, not necessarily the "optimistic" solution.

The outline of this work is as follows. The assumptions of our model are described in Section 2, where we present the ingredients of our model in a deterministic setting. Section 3 deals with the suggested reformulation technique for the bilevel problem. In Section 4, we show how our models can be adapted to handle uncertainty. We also show how our suggested reformulation can be adapted to this new setting. We assess our models and methodology in Section 5 on a case study built from realistic data. Some of the details of the model underlying the case study are provided in Appendix A. Finally, we conclude the paper in Section 6.

\section{The problem}

\subsection{Description}

In this paper we propose a stylized model wherein the current electrical system has transmuted into a likely potential future. In this future, several nearly independent subsystems, - micro-grids -, exist and interact with classical generation companies. We will make the assumption that micro-grids typically dispose of a set of generation assets mostly comprised of renewable intermittent sources (wind, solar). Remaining energetically independent of the rest of the system is then possible, up to a certain extent, by also disposing of a set of batteries. The remaining time, i.e., in case of production surplus or lack of generation, an interaction with the classical generators will provide useful back up to meet the total demand in energy. In our model we will focus on this last type of interaction from an abstract level and suggest several convenient reformulations so as to make the model tractable. This is non-trivial, since the formulated model will be a bilevel stochastic mixed integer optimization problem. Needless to say, the price to pay for tractability is a heuristic means of solving the problem, but by no means an unrealistic one. Indeed, our suggestion solution will be "feasible" ${ }^{1}$ for the original bilevel problem, but neither be the "optimistic" nor "pessimistic" solution. From the viewpoint of practice, this is actually not unrealistic since both extremes (the "optimistic" and "pessimistic" views) are stylized anyway. Indeed there is no reason to believe that an independent actor pursuing his own goals (e.g., in the case of micro-grids, this could be to remain independent energetically speaking most of the time), will purposely select the most advantageous response for the leader, nor any reason to believe it would select the most adverse option. Having something in between can thus be assumed reasonably realistic.

Before providing a more mathematical description of our model, let us begin by introducing the key elements of it.

Parties involved. We consider an extension of electricity production problems that involve two types of parties:

- GenCos are big producers of electricity in the network (they own large assets such as nuclear, thermal, hydro and other renewable energies). In order to avoid having to face an even tougher class of problems (an equilibrium problem with equilibrium constraints, or a coupled set of MPECs, e.g., [31]), we focus on the decision of a single operator that faces competing companies that have a fixed, perfectly known, policy. Hence, in what follows, GenCo refers to that single operator.

\footnotetext{
${ }^{1}$ Feasible here means that the given solution satisfies both upper and lower level constraints. Moreover at the given fixed upper level variables, the lower level variables are optimal for the lower level problem.
} 
- Each micro-grid $q \in Q$ consists of a small subnetwork that has highly volatile generation capacities (solar, wind), and two types of demands to be attended, which we denote as hard and elastic demands. On the one hand, hard demands must be met strictly at all times. On the other hand, elastic demands (heating up water, recharging electric cars, ...) can be shifted within a certain time window. This models the fact that environmentally aware users may be willing to postpone / anticipate an essential electrical consumption in order to reduce overall environmental burden (here translated through the system cost). Since this use is essential it will take place somewhere else within a given time window. Micro-grids are assumed to be relatively autonomous in terms of energy. However, due to the uncertain nature of their production, they need to buy or sell electricity from the GenCo.

Contract. Micro-grids and GenCos interact through contracts that specify the costs of buying/selling electricity from/to the GenCo for each period of the time horizon $T$. Specifically, each contract $k \in K$ is specified by (i) the price of contract $c_{k}$ paid by the micro-grid to the GenCo that proposes it, (ii) a linear cost function $x \mapsto f_{k t} x$ for buying the amount $x$ of electricity during time period $t$, and (iii) a linear function $y \mapsto g_{k t} y$ for selling the amount $y$ of electricity during time period $t$. We denote by $K_{0}$ the subset of contracts possibly proposed by the GenCo whose decisions are being optimized, while $K$ also contains contracts of competing companies.

Objective. On the one hand, the objective of the GenCo is to propose the least cost production schedule based on (i) the classical costs of unit-commitment and related problems (hydro optimization and nuclear maintenance scheduling, for instance) and (ii) the cost/benefit of buying/selling electricity to the microgrids. On the other hand, the objective of each micro-grid is to minimize its total cost of buying/selling electricity to the GenCos, by choosing a contract offered by the GenCo or one of the competing companies (in the latter case, the GenCo does not produce, buy or earn anything for/from the micro-grid). These two conflicting objectives can be naturally modeled as a bilevel optimization problem, introduced in the next section.

Coupling among the different time periods. We are given a time horizon $\mathcal{T}=\{1, \ldots, T\}$ that is further partitioned into days: $\mathcal{T}=D_{1} \cup \cdots \cup D_{J}$. Elastic loads couple all time periods of a given day. However, they do not couple time periods of different days as we may reasonably assume that the required load will be met during one full day. Nevertheless, time periods of different days may be coupled together in the presence of batteries with significant storage capacity. In this setting, the resulting multi-stage stochastic optimization problem is significantly harder, preventing us from decomposing the problem in time (by day). This is an immediate result of the temporal coupling of adjacent days through the large storage capacity batteries and the "strongly increasing complexity" of multi-stage stochastic programs with the number of stages (e.g., [32]).

\subsection{A bilevel formulation}

We describe below our mathematical model, represented as a bilevel multi-stage stochastic program. Let $x \in \mathbb{R}^{Q \times T}$ represent the electricity production of the GenCo. We are mainly interested here in the interaction between the GenCo and the micro-grids, so the value $x_{q t}$ represents the electricity produced by the GenCo and fed into micro-grid $q$ during period $t$. Similarly, we can define $y_{q t}$ as the amount of electricity bought by the GenCo from micro-grid $q$ during time period $t$. We denote by $F: \mathbb{R}^{Q \times 2 T} \rightarrow$ $\mathbb{R} \cup\{\infty\}$ the cost of producing $x-y$. Hence, one can think of the problem

$$
\min _{x, y \geq 0} F(x, y)
$$


as a compact abstract representation for the combination of unit-commitment, nuclear power plant maintenance planning, hydro power generation and other related problems, feeding the connected microgrids with the power described by $x-y$. The mapping $F$ can take the value $\infty$ for given vectors $x, y$ if certain constraints cannot be met. For instance, whenever $x-y$ exceeds the generation capacity of the system. With this convention any constraints on generation (or other constraints) can be readily incorporated in the framework by adding the characteristic function of these constraints to $F$. Let us note that computing $F(0,0)$ amounts to solving the power generation problem without considering microgrids. In particular, computing $F$ is therefore as difficult as computing the least cost power generation schedule. We also remark that in view of the above discussion, assuming that the GenCo problem is feasible without micro-grids means that $F(0,0)<\infty$. We note that from a computational viewpoint it is better to incorporate constraints directly in the model so as to avoid having to deal with very large numerical values. This is what is done in the numerical experiments. Handling constraints by allowing $F$ to take on the value $\infty$ is just convenient for the mathematical presentation.

In our model the vectors $x$ and $y$ indicate the power flow between the micro-grids and the (GenCo). Within the model it is not optimal to buy and sell energy at the same time. Hence at any optimal solution, $x$ and $y$ will not both be positive. Moreover, these variables are related to the internal functioning of the micro-grids and in particular their internal constraints. To this end we introduce an abstract constraint set $\mathcal{M}_{q}$ for each $q \in Q$, modelling in particular the demand-power balance. These power flows induce a certain cost governed by a contract. In our model, the GenCo has to decide which contracts it proposes to the micro-grids. We introduce an additional binary variable $Z_{q k}$ that is equal to 1 if contract $k$ is offered to micro-grid $q$ and zero otherwise. The index set $K$ will denote the set of all contracts and $K_{0} \subseteq K$, the set of contracts suggested by the GenCo, i.e., $K \backslash K_{0}$ is the set of contracts suggested by the competitors of the GenCo. We denote the set of contracts that the GenCo can offer as $\mathcal{Z} \subseteq\{0,1\}^{|Q| \times\left|K_{0}\right|}$. For instance, $\mathcal{Z}$ could contain all binary vectors, where the GenCo offers a fixed number of contracts $N_{q}$ to each micro-grid $q \in Q$. In that case, we would have $\mathcal{Z}=\left\{Z \in\{0,1\}^{|Q| \times\left|K_{0}\right|}: \sum_{k \in K_{0}} Z_{q k} \geq N_{q}, \forall q \in Q\right\}$.

For each micro-grid $q \in Q$, the binary variable $z_{q k}$ indicates if micro-grid $q$ subscribes to contract $k$. Notice that micro-grid $q$ interacts with the GenCo only if it subscribes to one of its contracts, that is, if $\sum_{k \in K_{0}} z_{q k} \geq 1$. We will introduce local versions of the variables $x, y$, called $\tilde{x}$ and $\tilde{y}$ that describe locally the power status. In particular, $x=\tilde{x}$ and $y=\tilde{y}$ only if a contract with the GenCo is subscribed. Summarizing, the deterministic bilevel problem below considers the following optimization variables:

- $Z_{q k}: 1$ if contract $k$ is offered to micro-grid $q$ (leader)

- $z_{q k}: 1$ if contract $k$ is subscribed by micro-grid $q$ (follower)

- $\tilde{x}_{q t}$ : power consumed by micro-grid $q$ during period $t$ (follower)

- $\tilde{y}_{q t}$ : power produced by micro-grid $q$ during period $t$ (follower)

- $x_{q t}$ : power consumed by micro-grid $q$ during period $t$ from the GenCo (follower)

- $y_{q t}$ : power produced by micro-grid $q$ during period $t$ for the GenCo (follower) 
We are now in measure to formulate our bilevel program:

$$
\begin{aligned}
& \min \quad F(x, y)-\sum_{q \in Q} \sum_{k \in K_{0}} z_{q k}\left(c_{k}+\sum_{t \in \mathcal{T}}\left(f_{k t} x_{q t}-g_{k t} y_{q t}\right)\right) \\
& \text { s.t. } \quad Z \in \mathcal{Z} \\
& \left(x_{q}, y_{q}, z_{q}\right) \in \underset{z_{q} \in\{0,1\}^{K}, \tilde{x}_{q}, \tilde{y}_{q}}{\arg \min } \sum_{k \in K} z_{q k}\left(c_{k}+\sum_{t \in \mathcal{T}}\left(f_{k t} \tilde{x}_{q t}-g_{k t} \tilde{y}_{q t}\right)\right), \quad \forall q \in Q \\
& \text { s.t. }\left(\tilde{x}_{q}, \tilde{y}_{q}\right) \in \mathcal{M}_{q}, \\
& x_{q}=\tilde{x}_{q} \sum_{k \in K_{0}} z_{q k}, \\
& y_{q}=\tilde{y}_{q} \sum_{k \in K_{0}} z_{q k}, \\
& z_{q k} \leq Z_{q k}, k \in K \\
& \sum_{k \in K} z_{q k}=1,
\end{aligned}
$$

where the constraints (2b), (3b)-(3f) are as explained below. The objective function of the leader, (2a), minimizes the production cost already mentioned in (1) combined with transaction costs related to the micro-grids. Similarly, the objective functions of the micro-grids, (3a), amounts to minimizing their total transaction costs with respect to all GenCos, not only the one represented by the leader. Hence, (3a) involves all contracts in $K$ while (2a) considers only the contracts in $K_{0}$. Constraints $(2 \mathrm{~b})$ and $(3 \mathrm{~b})$ restrain, respectively, the set of contracts offered by the GenCo and the feasible power consumption/production for the micro-grids. Constraints (3c) and (3d) model that $x_{q}=\tilde{x}_{q}$ and $y_{q}=\tilde{y}_{q}$ if and only if micro-grid $q$ subscribes to one of the contracts offered by the GenCo. Constraints (3e) impose that only the contract offered by the GenCo can be subscribed by the micro-grid, while constraints (3f) forces each micro-grid to select exactly one contract.

\section{MILP reformulations}

In this section we will provide two reformulations of the bilevel problem (2)-(3). In order to understand these, it is to be observed that problem (2)-(3) is not "unequivocally" defined in the sense that it is not clear what is meant with min in $(2 \mathrm{a})$, unless $\left(x_{q}, y_{q}, z_{q}\right)$ in $(3 \mathrm{a})$ is always unique. A classic way to remove this ambiguity is to consider a so called optimistic formulation wherein among all $\left(x_{q}, y_{q}, z_{q}\right)$ in the lower level problem (3) optimal set, the most advantageous for the leader is chosen. The pessimistic formulation then corresponds to picking the most disadvantageous case. Obviously both notions coincide whenever the lower level problem has a unique solution. We refer to [5] for further information on this topic. It is generally recognized that the pessimistic formulation is significantly harder to analyze than the optimistic formulation. In section 3.1 we will suggest a reformulation that is neither optimistic nor pessimistic, but somewhere in between. We also suggest a formulation leading to optimistic solutions in section 3.2 for comparative reasons. In particular the numerical experiments carried out in section 5 will show that results from both reformulations differ, hence implying that the $\arg \min$ in $(3 a)$ is indeed not unique and justifying that care should be taken in the interpretation. 


\subsection{Heuristic reformulation}

The bilinear optimization problem described in the previous section involves binary variables and nonlinear constraints, all that being built on the top of the already difficult optimization problem (1). Hence, we address the problem heuristically rather than exactly and we show below how it is possible to exploit the somewhat simple linking constraints (3e) to provide a heuristic reformulation for the bilinear problem.

We propose below a one-level reformulation of the bilevel problem that may lead to solutions that are not solutions to the optimistic problem. The key aspect of our reformulation relies on pre-processing. Specifically, for each $k \in K$ and $q \in Q$, we solve the restricted follower problem where $z_{q k}$ is equal to 1 , namely:

$$
\begin{array}{ll}
\min _{\tilde{x}_{q}, \tilde{y}_{q}} & c_{k}+\sum_{t \in \mathcal{T}}\left(f_{k t} \tilde{x}_{q k t}-g_{k t} \tilde{y}_{q k t}\right) \\
\text { s.t. } & \left(\tilde{x}_{q k}, \tilde{y}_{q k}\right) \in \mathcal{M}_{q} .
\end{array}
$$

We assume that the set of contracts and micro-grids is of reasonable cardinality so that this preprocessing step remains tractable. Let $\left(\bar{x}_{q k}, \bar{y}_{q k}\right)$ be an optimal solution of the above problem and $\bar{C}_{q k}$ be its solution cost. Then, a heuristic solution to the bilevel problem (2)-(3), which ensures the optimality of the lower level, can be found by solving:

$$
\begin{array}{ll}
\min _{z, Z} & F(x, y)-\sum_{q \in Q} \sum_{k \in K_{0}} \bar{C}_{q k} z_{q k} \\
\text { s.t. } & x_{q t}=\sum_{k \in K_{0}} \bar{x}_{q k t} z_{q k}, \quad \forall q \in Q, t \in \mathcal{T} \\
& y_{q t}=\sum_{k \in K_{0}} \bar{y}_{q k t} z_{q k}, \quad \forall q \in Q, t \in \mathcal{T} \\
& z_{q k} \leq Z_{q k}, \quad \forall q \in Q, k \in K \\
& \sum_{k \in K} z_{q k}=1, \quad \forall q \in Q \\
& z_{q k} \leq 1-Z_{q \ell}, \quad \forall k \in K, l \in K, q: \bar{C}_{q k}>\bar{C}_{q l} \\
& Z \in \mathcal{Z} \\
& z \in\{0,1\}
\end{array}
$$

The objective (4a) is obtained from (2a) by replacing the micro-grid cost for the GenCo by $\bar{C}_{q k} z_{q k}$. Similarly, (4b) and (4c) are obtained from (3c) and (3d) by replacing the variables $\tilde{x}$ and $\tilde{y}$ with their fixed values $\bar{x}$ and $\bar{y}$ computed in the pre-processing phase. It is exactly in these constraints that lies the heuristic aspect of our reformulation since the followers no longer choose the optimal solution that most benefits the leader, but instead take the one computed in the pre-processing phase, when (3a) admits several solutions. Finally, constraint (4f) ensures that the micro-grids choose the contracts that lead to the cheapest solutions. Indeed if $Z_{q l}=1$ and the cheap solution $\bar{C}_{q l}$ is available, any costlier solution is ruled out by equation (4f).

\subsection{Comparative exact formulation}

To assess the quality of our heuristic approach, we have also devised an exact one-level reformulation for the problem where (4b) and (4c) are replaced by non-linear constraints involving the aforementioned 
decision variables $\tilde{x}$ and $\tilde{y}$ that are restricted by the sets $\mathcal{M}_{q}$ for each $q \in Q$. In addition, the formulation contains restrictions enforcing $\tilde{x}$ and $\tilde{y}$ to be optimal for the subproblems.

$$
\begin{aligned}
\min _{z, Z, \tilde{x}, \tilde{y}} & F(x, y)-\sum_{q \in Q} \sum_{k \in K_{0}} \bar{C}_{q k} z_{q k} \\
\text { s.t. } & (4 \mathrm{~d})-(4 \mathrm{~g}) \\
& x_{q t}=\sum_{k \in K_{0}} \tilde{x}_{q k t} z_{q k}, \quad \forall q \in Q, t \in \mathcal{T} \\
& y_{q t}=\sum_{k \in K_{0}} \tilde{y}_{q k t} z_{q k}, \quad \forall q \in Q, t \in \mathcal{T} \\
& \bar{C}_{q k}=c_{k}+\sum_{t \in \mathcal{T}}\left(f_{k t} \tilde{x}_{q k t}+g_{k t} \tilde{y}_{q k t}\right), \quad \forall q \in Q, \forall k \in K \\
& \left(\tilde{x}_{q k}, \tilde{y}_{q k}\right) \in \mathcal{M}_{q} \\
& z \in\{0,1\}^{Q \times K}
\end{aligned}
$$

Constraints (5b) and (5c) contain bilinear terms that can be linearized using classical techniques. Specifically, let us introduce variables $X_{q k t}$ and $Y_{q k t}$, respectively equal to products $\tilde{x}_{q k t} z_{q k}$ and $\tilde{y}_{q k t} z_{q k}$. These variables can be substituded in constraints (5b) and (5c), adding also the constraints

$$
\begin{aligned}
& X_{q k t} \leq \tilde{x}_{q k t} \\
& X_{q k t} \leq M z_{q k} \\
& X_{q k t} \geq \tilde{x}_{q k t}-M\left(1-z_{q k}\right) \\
& Y_{q k t} \leq \tilde{y}_{q k t} \\
& Y_{q k t} \leq M z_{q k} \\
& Y_{q k t} \geq \tilde{y}_{q k t}-M\left(1-z_{q k}\right),
\end{aligned}
$$

that models the products through linear constraints involving big- $M$ coefficients. The difficulty of the resulting MILP is that big- $M$ coefficients often lead to numerical instability and weak LP relaxations. In our numerical experiments we have observed no specific difficulties related to the big-M coefficients.

\section{Stochastic extension of the model}

Power generation problems that involve renewable intermittent energy like wind and solar are subject to uncertainty, since the output of the renewable power plants depends on the weather conditions. This power output is therefore only partially known and should in principle be considered uncertain. We will set up a model in this section that accounts for uncertainty in generation. The resulting bilevel stochastic optimization problem will again become tractable due to a reformulation akin to the one used in the deterministic case. Obviously the main impact of considering uncertainty is that the preprocessing step will become much harder.

\subsection{Model}

The most important assumption for our stochastic model below is how we set up the structure of information. The main assumption, making a reformulation as in the deterministic case feasible, is that 
we will restrict the choice of contracts to the first stage. This means that all contracts have to be decided prior to observing any uncertainty. The second choice involves the structure of information within the remaining stochastic program. Therein we will assume that decent weather forecasts are available for an upcoming day and hence that all information for a given day is known at the beginning of that day. The stochastic program therefore has as many stages as there are days. In what follows we will denote with $\xi_{j}$ the uncertainty related to day $j \in\{1, \ldots, J\}$. Let us summarize these assumptions:

\section{- First stage}

- The GenCo chooses a set of contracts that are compatible with its objective.

- Each micro-grid chooses a contract among the contracts offered by the GenCos.

- Subsequent stages The exact productions and demands are known for all entities for all time periods that belong to the current day. Hence, the GenCo can produce the electricity and the micro-grids manage their elastic loads and batteries according to the chosen contracts and generated power.

A second important assumption that we will make is that uncertainty $\xi$ has finite support. It can therefore be represented by a finite set of scenarios $\Xi$ and uncertainty can be assumed to be represented by a scenario tree. For any given scenario $\xi \in \Xi$, we define $x(\xi)$ and $y(\xi)$ as the scenario dependent power flows between the GenCo and micro-grids. Following e.g., [33], we choose to not make explicit the non-anticipativity constraints which can be appended to the model through an additional abstract subspace to which the decisions should belong. Obviously on the scenario tree these conditions are readily enforced. As stated earlier, the optimization problem has as many stages as days and all information within a day is assumed to be known.

The introduction of uncertainty affects both the objective function and the constraints of the follower problem, as well as the objective function of the leader. Thanks to our specific measurability assumption concerning the days, uncertainty in constraints has an easy interpretation. In particular the abstract set $\mathcal{M}_{q}(\xi)$ (covering for instance power balance equations) is such that $\mathcal{M}_{q}(\xi)=\prod_{j=1}^{J} \mathcal{M}_{q}\left(\xi_{j}\right)$. Consequently, we can write $\left(\tilde{x}_{q}(\xi), \tilde{y}_{q}(\xi)\right) \in \mathcal{M}_{q}(\xi)$ a.s., which for a random realization at day $j$, simply means that $\left(\tilde{x}_{q}\left(\xi_{j}\right), \tilde{y}_{q}\left(\xi_{j}\right)\right) \in \mathcal{M}_{q}\left(\xi_{j}\right)$ as a plain deterministic constraint.

As uncertainty is also present in the objective function, this calls for a consideration of a tradeoff between risk and average performance (see e.g., [34] for a thorough discussion on such matters). As is common in practice, we will adopt a Markowitz type of idea so that we will weigh a measure of risk and average performance. To this end we will endow ourselves with a convex weight $\lambda$, that will weigh the average performance and the risk measure for the leader (the micro-grids are assumed risk-neutral for now, we discuss how to relax this assumption later). We choose to use the $\mathrm{CVaR}_{\epsilon}$ as a measure of risk as it preserves convexity and moreover allows for a linear reformulation due to the following characterization (e.g., $[35])$ :

$$
\operatorname{CVaR}_{\epsilon}[X(\xi)]=\min _{v}\left\{v+\frac{1}{1-\epsilon} \mathbb{E}\left[(X(\xi)-v)^{+}\right]\right\}
$$

for any random variable $X$ and probability level $\epsilon \in(0,1)$. 
We can now present the following risk-averse version of the bilevel energy management problem:

$$
\begin{aligned}
& \min \lambda \mathbb{E}\left[F(x(\xi), y(\xi))-\sum_{q \in Q} \sum_{k \in K_{0}}\left(c_{k}+\sum_{t \in \mathcal{T}}\left(f_{k t} x_{q t}(\xi)-g_{k t} y_{q t}(\xi)\right)\right) z_{q k}\right] \\
& +(1-\lambda) \operatorname{CVaR}_{\epsilon}\left[F(x(\xi), y(\xi))-\sum_{q \in Q} \sum_{k \in K_{0}}\left(c_{k}+\sum_{t \in \mathcal{T}}\left(f_{k t} x_{q t}(\xi)-g_{k t} y_{q t}(\xi)\right)\right) z_{q k}\right] \\
& \left(x_{q}(\xi), y_{q}(\xi), z_{q}\right) \in \underset{z_{q} \in\{0,1\}^{K}, \tilde{x}_{q}, \tilde{y}_{q}}{\arg \min } \mathbb{E}\left[\sum_{k \in K}\left(c_{k}+\sum_{t \in \mathcal{T}}\left(f_{k t} x_{q t}(\xi)-g_{k t} y_{q t}(\xi)\right)\right) z_{q k}\right], \quad q \in Q \\
& \text { s.t. }\left(\tilde{x}_{q}(\xi), \tilde{y}_{q}(\xi)\right) \in \mathcal{M}_{q}(\xi) a . s ., \quad \xi \in \Xi \\
& x_{q}(\xi)=\tilde{x}_{q}(\xi) \sum_{k \in K_{0}} z_{q k}, \quad \xi \in \Xi \\
& y_{q}(\xi)=\tilde{y}_{q}(\xi) \sum_{k \in K_{0}} z_{q k}, \quad \xi \in \Xi \\
& z_{q k} \leq Z_{q k}, \quad k \in K_{0}, \xi \in \Xi \\
& \sum_{k \in K} z_{q k}=1, \quad \xi \in \Xi
\end{aligned}
$$

\subsection{Heuristic reformulation}

As in the deterministic case, the problem (8)-(9) can be solved heuristically by solving follower problems in a pre-processing phase. For each $k \in K$ and $q \in Q$, we solve the restricted follower problem where $z_{q k}$ is equal to 1

$$
\begin{aligned}
\min _{\tilde{x}_{q}(\xi), \tilde{y}_{q}(\xi)} & c_{k}+\mathbb{E}\left[\sum_{t \in \mathcal{T}}\left(f_{k t} \tilde{x}_{q t}(\xi)-g_{k t} \tilde{y}_{q t}(\xi)\right)\right] \\
\text { s.t. } & \left(\tilde{x}_{q}(\xi), \tilde{y}_{q}(\xi)\right) \in \mathcal{M}_{q}(\xi) a . s ., \quad \forall \xi \in \Xi,
\end{aligned}
$$

under non-anticipativity constraints. Let $\left(\bar{x}_{q k}, \bar{y}_{q k}\right)$ be an optimal solution and $\overline{\mathbb{C}}_{q k}$ be its optimal value, namely,

$$
\overline{\mathbb{C}}_{q k}=c_{k}+\mathbb{E}\left[\sum_{t \in \mathcal{T}}\left(f_{k t} \bar{x}_{q t}(\xi)+g_{k t} \bar{y}_{q t}(\xi)\right)\right] .
$$


Then, a heuristic solution to the stochastic bilevel problem (8)-(9), which ensures the optimality of the lower level, can be found by solving:

$$
\begin{array}{ll}
\min _{z, Z} \quad \lambda \mathbb{E}\left[F(x(\xi), y(\xi))-\sum_{q \in Q} \sum_{k \in K_{0}}\left(c_{k}+\sum_{t \in \mathcal{T}}\left(f_{k t} x_{q t}(\xi)-g_{k t} y_{q t}(\xi)\right)\right) z_{q k}\right] \\
& +(1-\lambda) \operatorname{CVaR}_{\epsilon}\left[F(x(\xi), y(\xi))-\sum_{q \in Q} \sum_{k \in K_{0}}\left(c_{k}+\sum_{t \in \mathcal{T}}\left(f_{k t} x_{q t}(\xi)-g_{k t} y_{q t}(\xi)\right)\right) z_{q k}\right] \\
\text { s.t. } \quad & z_{q k} \leq Z_{q k}, \quad \forall q \in Q, k \in K_{0} \\
& \sum_{k \in K} z_{q k}=1, \quad \forall q \in Q \\
& x_{q t}(\xi)=\sum_{k \in K_{0}} \bar{x}_{q k t}(\xi) z_{q k}, \quad \forall q \in Q, t \in \mathcal{T}, \xi \in \Xi \\
& y_{q t}(\xi)=\sum_{k \in K_{0}} \bar{y}_{q k t}(\xi) z_{q k}, \quad \forall q \in Q, t \in \mathcal{T}, \xi \in \Xi \\
& z_{q k} \leq 1-Z_{\ell q}, \quad \forall k, q: \overline{\mathbb{C}}_{q k}>\overline{\mathbb{C}}_{q l} \\
& Z \in \mathcal{Z} \\
& z \in\{0,1\}^{Q \times K} .
\end{array}
$$

The above program can be further reformulated by replacing CVaR with its characterization (7) and by introducing the optimization variables $w(\xi)$ to linearize the function $[\cdot]^{+}$. This yields the regular stochastic program:

$$
\begin{aligned}
\min _{z, Z, w(\xi)} & \mathbb{E}\left[\lambda F(x(\xi), y(\xi))-\lambda \sum_{q \in Q} \sum_{k \in K_{0}}\left(c_{k}+\sum_{t \in \mathcal{T}}\left(f_{k t} x_{q t}(\xi)-g_{k t} y_{q t}(\xi)\right)\right) z_{q k}+(1-\lambda)\left(v+\frac{w(\xi)}{1-\epsilon}\right)\right] \\
\text { s.t. } & (11 \mathrm{~b})-(11 \mathrm{~h}) \\
& w(\xi) \geq F(x(\xi), y(\xi))-\sum_{q \in Q} \sum_{k \in K_{0}}\left(c_{k}+\sum_{t \in \mathcal{T}}\left(f_{k t} x_{q t}(\xi)-g_{k t} y_{q t}(\xi)\right)\right) z_{q k}-v, \quad \forall \xi \in \Xi \\
& w(\xi) \geq 0, \quad \forall \xi \in \Xi .
\end{aligned}
$$

We mention that a similar MILP reformulation can be obtained whenever the micro-grids are also assumed to be risk-averse. For instance, we could consider the same Markowitz type model and endow ourselves with a set of convex multipliers $\left\{\lambda^{q}\right\}_{q \in Q}$ weighting the expectation and $\mathrm{CVaR}_{\epsilon}$ for each microgrid. The resulting counterpart of (10) is more demanding computationally due to the difficulty of decomposing the problem per scenario. This difficulty is well-known in risk-averse stochastic optimization, see for instance the recent [33]. As this goes beyond the scope of this paper, we disregard this extension in what follows. 


\subsection{Comparative reformulation}

As in the deterministic case, the above heuristic reformulation can be made exact by replacing constraints (11d) and (11e) with the bilinear constraints

$$
\begin{aligned}
& x_{q t}(\xi)=\sum_{k \in K_{0}} \tilde{x}_{q k t}(\xi) z_{q k}, \quad \forall q \in Q, t \in \mathcal{T}, \xi \in \Xi \\
& y_{q t}(\xi)=\sum_{k \in K_{0}} \tilde{y}_{q k t}(\xi) z_{q k} \forall q \in Q, t \in \mathcal{T}, \xi \in \Xi
\end{aligned}
$$

and adding constraints

$$
\begin{aligned}
& \overline{\mathbb{C}}_{q k}=c_{k}+\mathbb{E}\left[\sum_{t \in \mathcal{T}}\left(f_{k t} \tilde{x}_{q t}(\xi)+g_{k t} \tilde{y}_{q t}(\xi)\right)\right], \quad \forall q \in Q, k \in K \\
& \left(\tilde{x}_{q k}(\xi), \tilde{y}_{q k}(\xi)\right) \in \mathcal{M}_{q}(\xi), \quad \forall q \in Q, k \in K, \xi \in \Xi .
\end{aligned}
$$

Moreover optimization is now to be carried out over $(z, Z, \tilde{x}(\xi), \tilde{y}(\xi))$. Evidently the resulting problem is significantly harder!

\section{Case study based on thermal power unit-commitment}

We assess below our heuristic reformulation on a power generation problem where $F$ represents a thermal unit-commitment problem, following closely [36]. The latter minimizes the total operation cost, which is defined as the sum of the production cost, the startup cost, and the shutdown cost. The production cost is piece-wise linear, while the startup cost is piece-wise linear approximation of an exponential function of the off-line time prior to the startup. Since the MILP model is rather technical, and because computing $F(x, y)$ is not the focus of this work, we refer the interested reader to, e.g., [36] for the details about the mixed-integer linear program behind $F$. We also refer to $[1,2]$ and the many references therein for further alternatives to the model considered here. In addition, we detail the constraints used in our micro-grid model in Appendix A.

\subsection{Data}

\subsubsection{General system}

The time horizon is one week starting on Monday at $12 \mathrm{am}$, each day is split in periods of one hour, i.e., $|\mathcal{T}|=168$. The data used for the unit-commitment part is provided by [36], 50 generators are used giving a daily fixed load of $135,5 \mathrm{GWh}$.

\subsubsection{Considered contracts}

Several types of contracts are proposed combining the possibility of lower prices during nighttime and/or the possibility of lower prices during the weekend. This leads to a total of four types of contracts. The competitors offer one contract of each type to each micro-grid. The GenCo generates four possible contracts of each type, thus $\left|K_{0}\right|=16$ and $|K|=20$. For all contracts, the average cost of $1 \mathrm{~kW}$ is about $0,10 \mathrm{EUR}^{2}$.

\footnotetext{
${ }^{2}$ http://www.rte-france.com/fr/document/bilan-previsionnel-de-l-equilibre-offredemande-2016
} 


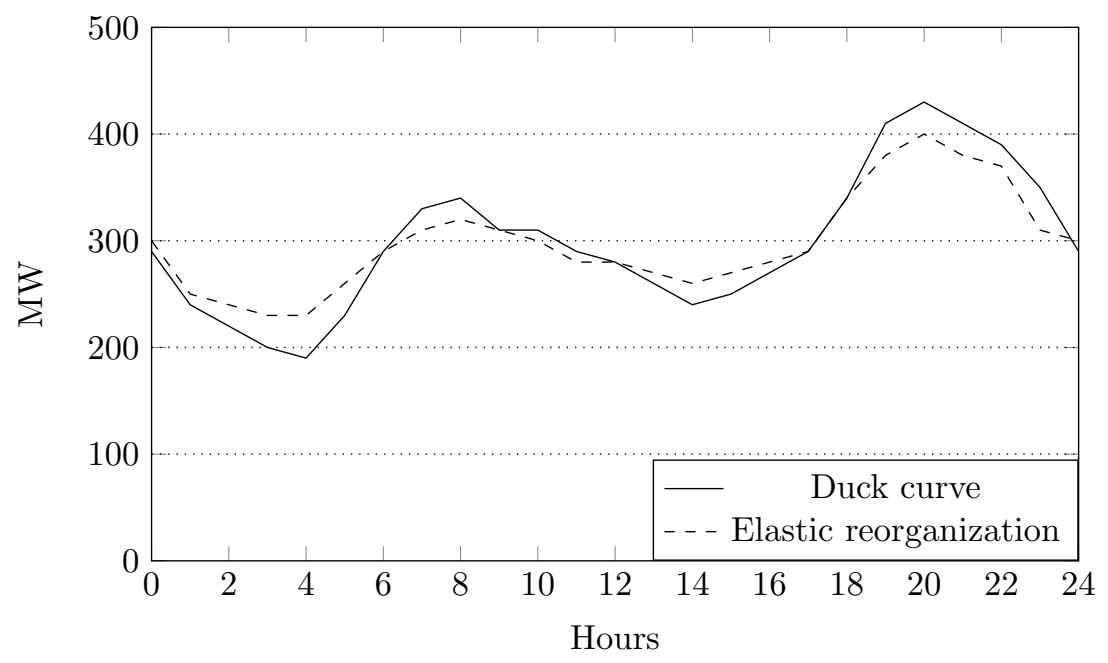

Figure 1: Duck curve example for a micro-grid with 10000 devices

\subsubsection{Description of the micro-grids}

In what follows we will define devices as an abstract group of objects fulfilling a purpose of consumption, production or storage under time constraints within a micro-grid. The devices considered, their consumption and the time periods where they are used fit numbers reported in a recent study of electricity demand/offer equilibrium in France ${ }^{2}$.

- Consumption devices are devices appearing in a common household, which we split into three further sub-classes:

- Constant: these devices consume power constantly. They represent $30 \%$ of the daily consumption.

- Comfort: devices that consume power during a predetermined period each day, representing $30 \%$ of the daily consumption.

- Elastic: devices for which predetermined windows of usage are defined for each day as well as a total daily load. They represent $40 \%$ of the daily consumption.

The periods where comfort devices are used and elastic devices can be used as well as their consumptions are randomly generated such that the graph of the total consumption of a micro-grid fits a classical duck curve for each day. The average consumption of 200 devices over one week is $1 \mathrm{GWh}$, which corresponds to the consumption of 5000 common (European) households. Consumption of elastic devices can be reorganized to reduce costs by taking advantage of low hourly prices, production devices and storage devices. Figure 1 illustrates the hourly demand of the micro-grid composed of 10000 devices.

- Production devices are equally split into solar panels and wind turbines. The production capacity of each production device is generated randomly. For each micro-grid, their production capacity is sufficient to satisfy on average $50 \%$ of the consumption of micro-grids. 
- Storage devices are of a single type, their capacity is sufficient to store on average $30 \%$ of the daily electricity load of a micro-grid. We consider that during the storage process there is a total loss of $10 \%$ of energy due to physical constraints which corresponds to the storage efficiency of state-of-the-art domestic batteries such as Tesla's Powerwall ${ }^{3}$.

In the largest instance, 90,000 devices are considered, corresponding to over 2, 000,000 households. The total consumption of devices in this instance is on average $64 \mathrm{GWh}$ per day. As on average $50 \%$ of the daily load is supplied by production devices, about 32 GWh must be provided from the global network each day.

The penetration of the MGs in the total load of the GenCo is defined as

$$
\text { penetration }=\frac{\text { load of the } M G s}{\text { total load for the GenCo }}
$$

If all MG considered choose a contract from the GenCo, the penetration rate is about $19 \%$.

\subsubsection{Uncertainty}

Four simple independent scenarios are considered based on the possibility of good sunshine and good wind that influence production of micro-grids. Weather conditions are considered as identical each day of the week in a scenario. In bad conditions (no sunshine or no wind), a production device produces only $50 \%$ of its maximum production capacity. Notice that this implies that the multi-stage structure of the problem is simplified to a two-stage problem for which no non-anticipativity constraints are required. The value of $\epsilon$ used in CVaR is equal to 0.1, representing a high aversion to risk. The full instance details are available from the authors upon request.

\subsection{Numerical results}

All the methods were implemented using Julia 0.4.6 and ILOG CPLEX 12.7 as MIP solver. Tests were made on a 4-core i7 $2.30 \mathrm{GHz}$ processor with 16Go of RAM memory. Maximum computation time is set to 3600 s.

Table 1 presents the average computation times for solving the follower problems. Times and objectives are averaged over the 20 possible contracts for the 5 instances of micro-grids of each size. The objectives are the average consumption costs for micro-grids of a given size which is also the average income for a GenCo supplying those micro-grids. They are provided in euros. The standard deviation $(\sigma)$ of the objective value is also provided. These results show that solving the restricted follower problem can be done fairly quickly. The objective value for all contracts is positive and for all instances considered, there exists at least one contract of the GenCo that is cheaper and one that is more expensive than those of the competitors. Each micro-grid is financially interesting for the GenCo if the additional production costs are not too high. In terms of size, the formulation of the follower problem on a micro-grid with 100 devices and a single contract available contains 59136 variables and 35390 constraints.

Figure 2 illustrates the optimal demand strategies over time of two micro-grid of 5000 devices for two contracts. The left graph represents a micro-grid composed of households and the right one a micro-grid composed of offices. One contract considers constant buying and selling prices over time (full line) and the other considers smaller prices during nighttime and weekends (dashed line). The different demand strategies will influence the production of the GenCo if the two contracts are proposed.

\footnotetext{
${ }^{3}$ https://www.tesla.com/fr_BE/powerwall?redirect $=$ no
} 


\begin{tabular}{|c|c|c|c|}
\hline \# Devices & Avg. Obj & $\sigma$ & time(s) \\
\hline 100 & 34.99 & 2.25 & 0.15 \\
200 & 37.41 & 3.67 & 0.32 \\
1000 & 207.51 & 14.97 & 1.91 \\
2000 & 332.93 & 21.32 & 3.95 \\
5000 & 653.61 & 37.36 & 14.57 \\
10000 & 957.65 & 40.56 & 24.01 \\
\hline
\end{tabular}

Table 1: Average computation time and objective value for the micro-grid power planning preprocessing step.
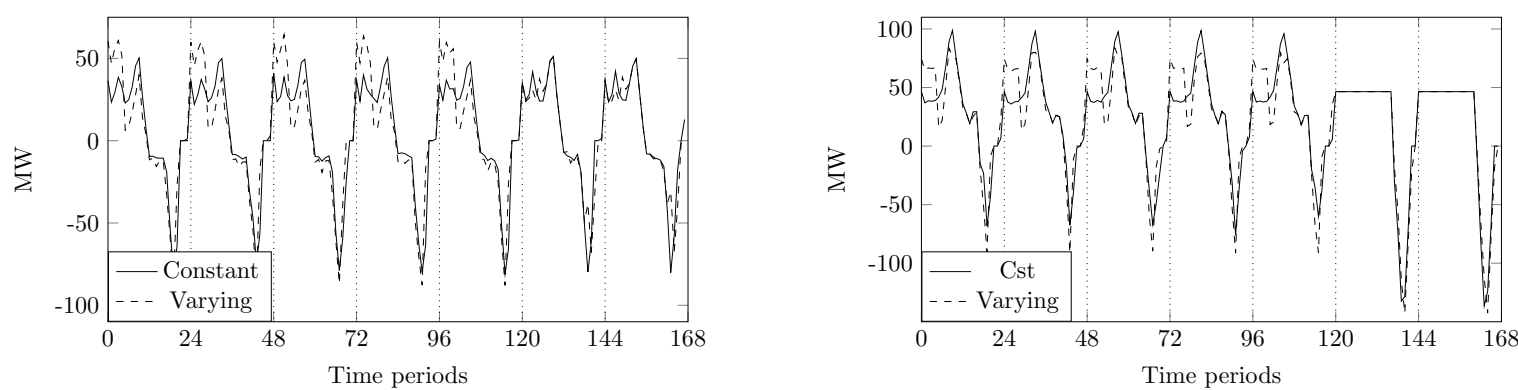

Figure 2: Micro-grid demand management

Notice that the curves have a different aspect than a classical duck curve illustrated in Figure 1. This is due to production devices as solar panel that can be active only during daytime. As a result, the demand is negative almost all daytime and reaches its biggest values during nighttime. The right hand side graph is mostly flat during the weekend as the micro-grid considered is composed of offices with very low consumption during this period.

Table 2 presents results solving small instances with the exact (Exact optimistic) and the heuristic (Heuristic) bilevel reformulations. Here, the value of $\lambda$ is set to 1 in order to optimize the expectation. The first two columns report the number of devices in the considered micro-grids and the number of micro-grids of each size. Hence, the first line considers one micro-grid with 100 devices and the last line considers five micro-grids with 100 devices plus five micro-grids with 200 devices.

It has been observed in the results that for each instance, both approaches (Heuristic and Exact) select the same contracts for each micro-grid.

The difference between optimal values lies in the extra production cost there is for the GenCo to satisfy the demand of the micro-grids who subscribed a contract. Specifically, in Exact, the planning of elastic devices of those micro-grids can be reorganized to reduce the cost of the UC problem, preserving optimal cost for the micro-grids. This observation shows that the bilevel problem (8)-(9) (or (2)-(3)) is indeed ambiguous in so much that the argmin of problem (9) is not unique. Consequently the pessimistic and optimistic versions of the model differ and our heuristic reformulation offers something in between. However, this gap, as reported in the column $\Delta(F-U C)$ is not very large. Indeed, let us denote by $\left(x_{o p t}, y_{o p t}\right)$ and $\left(x_{\text {heur }}, y_{\text {heur }}\right)$ the power generation returned by Exact and Heuristic, respectively. Columns $F\left(x_{\text {opt }}, y_{\text {opt }}\right)-F(0,0)$ and $F\left(x_{\text {heur }}, y_{\text {heur }}\right)-F(0,0)$ correspond to GenCo's extra production costs due to the consumption of the micro-grids in the solutions of Exact and Heuristic respectively. 


\begin{tabular}{|c|c|c|c|c|c|c|}
\hline \multicolumn{2}{|c|}{ Test set } & \multicolumn{2}{|c|}{ Exact } & \multicolumn{2}{c|}{ Heuristic } & \multirow{2}{*}{$\Delta(F-U C)(\%)$} \\
\hline \# Devices & $\mathrm{nb}$ & $F\left(x_{\text {opt }}, y_{\text {opt }}\right)-F(0,0)$ & time $(\mathrm{s})$ & $F\left(x_{\text {heur }}, y_{\text {heur }}\right)-F(0,0)$ & time $(\mathrm{s})$ & \\
\hline 100 & 1 & 8.134968 & 76.26 & 8.146005 & 26.9 & 0.136 \\
100 & 2 & 29.413995 & 202.3 & 29.438447 & 28.63 & 0.083 \\
100 & 3 & 67.440871 & 312.46 & 67.471015 & 28.07 & 0.045 \\
100 & 4 & 52.290345 & 678.9 & 52.334735 & 26.67 & 0.085 \\
100 & 5 & 24.543927 & 690.63 & 24.588317 & 27.22 & 0.181 \\
200 & 1 & 0 & 135.01 & 0 & 21.16 & 0.021 \\
200 & 2 & 71.09899 & 242.34 & 71.1142 & 25.88 & 0.022 \\
200 & 3 & 128.69007 & 635.02 & 128.717851 & 25.85 & 0.022 \\
200 & 4 & 188.672761 & 1684 & 188.715209 & 25.95 & 0.025 \\
200 & 5 & 171.455653 & 1919.43 & 171.498219 & 25.7 & 0.018 \\
$100-200$ & 1 & 60.079001 & 223.74 & 60.090038 & 25.93 & 0.041 \\
$100-200$ & 2 & 100.510032 & 893.82 & 100.550933 & 27.19 & 0.030 \\
$100-200$ & 3 & 196.12615 & 1697.23 & 196.184324 & 28.38 \\
$100-200$ & 4 & 240.957646 & 3520.43 & 241.045643 & 0.037 \\
$100-200$ & 5 & - & $>3600$ & 196.081488 & - \\
\hline
\end{tabular}

Table 2: Comparing Exact and Heuristic on small instances.

The value

$$
\Delta(F-U C)=\frac{F\left(x_{\text {heur }}, y_{\text {heur }}\right)-F\left(x_{\text {opt }}, y_{\text {opt }}\right)}{F\left(x_{\text {opt }}, y_{\text {opt }}\right)-F(0,0)}
$$

is the relative gap between the costs of extra production in Heuristic and Exact. The reported computation times of Heuristic do not include the preprocessing times.

Computation times of Heuristic (without considering preprocessing) are quite stable while those of Exact tend to grow very quickly and the last instance considering 10 micro-grids cannot be solved in one hour. Concerning the instance with a single micro-grid with 200 devices, the extra production cost is 0 because the micro-grid chose a contract of another company. The gap on extra production cost is very low in all tests. The Heuristic/Exact formulation of the instance with five micro-grids of 100 devices contains 200/300 binary variables, 1680/37255 continuous variables and 1975/279725 constraints. As no variables are related to devices in the Heuristic formulation, the number of devices does not impact its the size.

Table 3 reports the results of Heuristic on larger instances. We consider three values for $\lambda: 1,0.5$ and

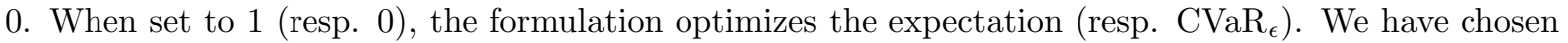
a value $\epsilon$ of 0.1 , representing a high aversion to risk. In each instance, five micro-grids of each size are used. Columns E report the expectation of the optimal solutions, columns CVaR report their conditional value-at-risk, both are given in thousands of $€$. Columns sold report the number of contracts of the GenCo chosen by the MG in the optimal solution found. These values are reported only for $\lambda=1$ as they were identical for other values of $\lambda$ after rounding. The first line considers only the original UC problem while the last line considers 20 micro-grids ( 5 of each size) with a maximum possible penetration of $19 \%$. Notice the negative solution cost of the last line, which is obtained from a revenue from the micro-grids that is higher than the production cost. Computation times tend to grow slowly when adding microgrids and stay close to the computation time of the original UC problem when $\lambda=1$. For $\lambda=0.5$, the expectation is similar, the CVaR decreases significantly for some instances and the computation time increases of about 20\%. With $\lambda=0$, results for the expectation and CVaR are similar than for $\lambda=0.5$ and computation times are more than doubled for most instances in comparaison of $\lambda=1$.

Our formulation does not increase much the difficulty of the UC problem on the tested instances when 


\begin{tabular}{|c|c|c|c|c|c|c|c|c|c|c|c|}
\hline \multirow{2}{*}{ Test set } & \multicolumn{5}{|c|}{$\lambda=1$} & \multicolumn{3}{|c|}{$\lambda=0.5$} & \multicolumn{3}{|c|}{$\lambda=0$} \\
\hline & $\mathrm{E}$ & CVaR & time $(\mathrm{s})$ & sold & penetration & $\mathrm{E}$ & CVaR & time $(\mathrm{s})$ & $\mathrm{E}$ & CVaR & time $(\mathrm{s})$ \\
\hline $\mathrm{UC}$ & 3599 & - & 20.98 & 0 & 0 & 3599 & - & 20.98 & 3599 & - & 20.98 \\
\hline 1000 & 3221 & 3580 & 26.31 & 5 & $1.2 \%$ & 3221 & 3250 & 30.09 & 3221 & 3250 & 47.41 \\
\hline 2000 & 2973 & 3304 & 22.98 & 4 & $2.1 \%$ & 2973 & 3018 & 26.37 & 2973 & 3018 & 46.71 \\
\hline 5000 & 1373 & 1526 & 24.38 & 5 & $6.0 \%$ & 1374 & 1510 & 27.33 & 1375 & 1509 & 46.54 \\
\hline 10000 & -421 & 768 & 24.98 & 5 & $10.9 \%$ & -421 & -144 & 29.87 & -420 & -145 & 56.54 \\
\hline $1000-2000$ & 2595 & 2884 & 25.00 & 9 & $3.3 \%$ & 2595 & 2670 & 28.09 & 2595 & 2670 & 50.53 \\
\hline $1000-2000-5000$ & 378 & 960 & 25.61 & 14 & $9.1 \%$ & 379 & 589 & 30.24 & 379 & 589 & 50.63 \\
\hline $1000-2000-5000-10000$ & -2055 & 145 & 32.42 & 10 & $12.6 \%$ & -2055 & -1768 & 44.19 & -2053 & -1769 & 82.29 \\
\hline
\end{tabular}

Table 3: Solution of Heuristic for larger instances.

minimizing the expectation. Integrating $\mathrm{CVaR}$ in the objective function with $\lambda=0.5$ seems interesting as it is not very time consuming and reduces $\mathrm{CVaR}$ almost to its best attainable value.

The number of contracts sold per instance reflects the quadratic aspect of production costs considered in the formulation for the UC [36]. Summing the numbers of contracts sold in instances where all microgrids have the same size (lines 2 to 5 of Table 3), a total of 19 contracts are sold to the 20 micro-grids. When solving the problem with all 20 micro-grids together (last line of the table), only 10 contracts are sold. In this solution, respectively 2, 1, 4 and 3 contracts are sold to the MGs of size 1000, 2000, 5000 and 10,000. The results of this last instance let us suppose the GenCo cannot afford having a penetration rate higher than about $13 \%$ with the contracts proposed by the competitors. Even the small MG with 1000 devices go to competitors illustrating that the GenCo can only propose contracts more expensive than those of competitors. If many micro-grids must be supplied with electricity, the average production costs increases for the GenCo in the UC problem. At some point, the GenCo cannot afford proposing low price contracts to additional micro-grids, otherwise it would produce at a loss. Our approach prevents the GenCo from falling in this situation by proposing contracts that are more expensive than those of the competitors to micro-grids that are not financially interesting.

For each instance, the same MGs choose a contract of the GenCo for each value of $\lambda$ considered but the contracts sold are not all identical. Table 4 illustrates the solution of the instance with 5 micro-grids of size 5000. The contracts are represented in the columns and are grouped by type. Constant price, daytime-nighttime price, week-weekend prices and daytime-nighttime plus week-weekend prices. A blank dot appears when a contract is proposed to a micro-grid by the GenCo, a black dot appears when a micro-grid selects a contract. The five micro-grids select a contract from the GenCo for all values of $\lambda$ but the contracts proposed and chosen for the fourth and fifth micro-grid vary. Integrating CVaR can discredit contracts that present a high risk in some scenarios.

\section{Conclusions and perspectives}

We have addressed a variant of power generation optimization problems where the GenCo interacts with micro-grids by buying or selling power to them. We have modeled the problem as a bilevel stochastic optimization problem, built on the top of the already difficult power generation optimization problem that the GenCo must solve to produce its energy. For realistic size data, solving the bilevel problem exactly is out of reach so that we have focused on a heuristic reformulation that produces a solution to the bilevel problem that is neither "optimistic" nor "pessimistic". Although our numerical results exhibit that the resulting solution is indeed different from the "optimistic" solution, they also indicated 


\begin{tabular}{|c|c|c|c|c|c|c|c|c|c|c|c|c|c|c|c|c|c|}
\hline \multicolumn{2}{|c|}{ Test set } & \multicolumn{4}{|c|}{ Cst price } & \multicolumn{4}{|c|}{ DN price } & \multicolumn{4}{|c|}{ WWE price } & \multicolumn{4}{|c|}{ DN and WWE price } \\
\hline $\mathrm{MG}$ & $\lambda$ & 1 & 2 & 3 & 4 & 5 & 6 & 7 & 8 & 9 & 10 & 11 & 12 & 13 & 14 & 15 & 16 \\
\hline \multirow{3}{*}{$\neg$} & 1 & 0 & & & & o & & 0 & & 0 & O & 0 & 0 & 0 & & 0 & $\bullet$ \\
\hline & 0.5 & ○ & & & & 0 & & 0 & & 0 & 0 & ० & 0 & 0 & & 0 & $\bullet$ \\
\hline & 0 & ○ & & & & ○ & & ○ & & ○ & ○ & ○ & 0 & O & & $\circ$ & $\bullet$ \\
\hline \multirow{3}{*}{ 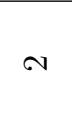 } & 1 & o & & & & o & & o & $\bullet$ & & o & o & 0 & & & 0 & \\
\hline & 0.5 & ○ & & & & ○ & & ० & $\bullet$ & & ० & ० & ० & & & $\circ$ & \\
\hline & 0 & 0 & & & & 0 & & 0 & $\bullet$ & & 0 & 0 & 0 & & & 0 & \\
\hline \multirow{3}{*}{$\infty$} & 1 & 0 & & & & o & & o & & o & o & o & 0 & 0 & & 0 & $\bullet$ \\
\hline & 0.5 & ० & & & & ○ & & ० & & o & ० & ० & 0 & O & & o & • \\
\hline & 0 & 0 & & & & 0 & & 0 & & 0 & 0 & 0 & 0 & 0 & & 0 & $\bullet$ \\
\hline \multirow{3}{*}{$\nabla$} & 1 & 0 & & & & 0 & & 0 & $\bullet$ & 0 & O & 0 & 0 & 0 & & 0 & 0 \\
\hline & 0.5 & 0 & & & & 0 & & O & • & ○ & 0 & ○ & 0 & 0 & & 0 & 0 \\
\hline & 0 & 0 & & & & 0 & & 0 & & 0 & 0 & 0 & 0 & 0 & & 0 & $\bullet$ \\
\hline \multirow{3}{*}{10} & 1 & 0 & & & & 0 & & 0 & 0 & • & 0 & 0 & 0 & 0 & 0 & 0 & 0 \\
\hline & 0.5 & O & • & & & o & & o & o & & 0 & O & 0 & $\circ$ & 0 & 0 & 0 \\
\hline & 0 & 0 & $\bullet$ & & & 0 & & o & o & & 0 & 0 & 0 & 0 & 0 & 0 & 0 \\
\hline
\end{tabular}

Table 4: Contracts proposed and selected to five MG with $|D|=5000$.

that the gap is small (roughly $0.05-0.1 \%$ variation on the upper level objective function). Interestingly enough, we could link this discrepancy between the optimistic bilevel solution and our solution to the existence of load-shifting devices within the micro-grid that are piloted differently in the "optimistic" situation.

We have assessed our reformulation on a case-study based on a thermal unit-commitment problem using realistic data. Our numerical results have confirmed the tractability of the heuristic approach, since the solution time of our reformulation is at most $50 \%$ higher than the one required for solving the thermal unit-commitment problem. In contrast, the solution of an exact reformulation of the bilevel problem increases the solution times by more than $10000 \%$ in the presence of only 8 micro-grids. Our results also confirm the quality of the approximation provided by our heuristic, which provides a solution very close to the solution of the exact "optimistic" model. An interesting venue for future research would seek to extend the kind of reformulations proposed in this paper to other bilevel optimization problems with integer variables, which is a class of optimization problems that are notoriously difficult to solve exactly.

Acknowledgments. The authors are grateful for the comments of two anonymous referees.

[1] M. Tahanan, W. van Ackooij, A. Frangioni, F. Lacalandra, Large-scale unit commitment under uncertainty: a literature survey, 4OR 13 (2) (2015) 115-171. doi:10.1007/s10288-014-0279-y.

[2] W. van Ackooij, I. Danti Lopez, A. Frangioni, F. Lacalandra, M. Tahanan, Large-scale unit commitment under uncertainty: an updated literature survey, Submitted preprint: available http://eprints.adm.unipi.it/2374/1/ UUC-Survey.pdf (2018) 1-57.

[3] R. Taktak, C. d'Ambrosio, An overview on mathematical programming approaches for the deterministic unit commitment problem in hydro valleys, Energy Systems 8 (1) (2017) 57-79. doi:10.1007/s12667-015-0189-x.

[4] V. L. de Matos, D. P. Morton, E. C. Finardi, Assessing policy quality in a multistage stochastic program for long-term hydrothermal scheduling, Annals of Operations Research 253 (2) (2017) 713-731. doi:10.1007/s10479-016-2107-6. URL http://dx.doi.org/10.1007/s10479-016-2107-6 
[5] S. Dempe, V. Kalashnikov, G. A. Pérez-Valdés, N. Kalashnykova, Bilevel Programming Problems: Theory, Algorithms and Applications to Energy Networks, Energy Systems, Springer-Verlag, 2015.

[6] F. Facchinei, J. S. Pang, Finite-Dimensional Variational Inequalities and Complementarity problems: Volume I, Springer Series in Operations Research, Springer, 2003.

[7] F. Facchinei, J. S. Pang, Finite-Dimensional Variational Inequalities and Complementarity problems: Volume II, Springer Series in Operations Research, Springer, 2003.

[8] R. Henrion, J. Outrata, T. Surowiec, Analysis of m-stationary points to an epec modeling oligopolistic competition in an electricity spot market, ESAIM: Control, Optimisation and Calculus of Variations 18 (2012) 295-317. doi:10.1051/cocv/2011003.

[9] D. Aussel, P. Bendotti, M. Pištěk, Nash equilibrium in a pay-as-bid electricity market: Part 1-existence and characterization., Optimization 66 (6) (2017) 1013-1025.

[10] D. Aussel, P. Bendotti, M. Pištěk, Nash equilibrium in a pay-as-bid electricity market: Part 2 - best response of a producer., Optimization 66 (6) (2017) 1027-1053.

[11] B. Heymann, A. Jofré, Mechanism design and auctions for electricity network, in: D. Aussel, C. Latitha (Eds.), Generalized Nash Equilibrium Problems, Bilevel Programming and MPEC, Forum for Interdisciplinary Mathematics, Springer Singapore, 2017, pp. 65-81.

[12] J. S. Pang, M. Fukushima, Quasi-variational inequalities, generalized nash equilibria, and multi-leader-follower games, Computational Management Science 2 (1) (2005) 21-56.

[13] J. S. Pang, M. Fukushima, Quasi-variational inequalities, generalized nash equilibria, and multi-leader-follower games, Computational Management Science 6 (3) (2009) 373-375.

[14] J. S. Pang, M. Razaviyayn, A. Alvarado, Computing b-stationary points of nonsmooth DC programs, Mathematics of Operations Research 42 (1) (2017) 95-118. doi:10.1287/moor.2016.0795. URL http://dx.doi.org/10.1287/moor.2016.0795

[15] W. van Ackooij, W. de Oliveira, DC programming techniques with inexact subproblems' solution for general DC programs, Submitted manuscript (2017) 1-27.

[16] B. F. Hobbs, S. K. Nelson, A nonlinear bilevel model for analysis of electric utility demand-side planning issues, Annals of Operations Research 34 (1) (1992) 255-274. doi:10.1007/BF02098182.

[17] E. G. Kardakos, C. K. Simoglou, A. G. Bakirtzis, Optimal offering strategy of a virtual power plant: A stochastic bi-level approach, IEEE Transactions on Smart Grids 7 (2) (2016) 794-806. doi:10.1109/TSG.2015.2419714.

[18] C. Cervilla, J. Villar, F. A. Campos, Bi-level optimization of electricity tariffs and pv distributed generation investments, in: European Energy Market (EEM), 2015 12th International Conference on the, 2015, pp. 1-5. doi:10.1109/EEM.2015.7216711.

[19] G. E. Asimakopoulou, A. L. Dimeas, N. D. Hatziargyriou, Leader-follower strategies for energy management of multimicrogrids, IEEE Transactions on Smart Grids 4 (4) (2013) 1909-1916.

[20] S. Dempe, J. Dutta, Is bilevel programming a special case of a mathematical program with complementarity constraints?, Mathematical Programming 131 (1) (2012) 37-48.

[21] L. Adam, R. Henrion, J. Outrata, On M-stationarity conditions in MPECs and the associated qualification conditions, Mathematical Programming (2017) 1-31doi:10.1007s/s10107-017-1146-3.

[22] A. Haurie, R. Loulou, G. Savard, A two-player game model of power cogeneration in new england, IEEE Transactions on Automatic Control 32 (9) (1992) 1451-1456.

[23] R. Fernández-Blanco, J. M. Arroyo, N. Alguacil, X. Guan, Incorporating price-responsive demand in energy scheduling based on consumer payment minimization, IEEE Transactions on Smart Grids 7 (2) (2016) 817-826. doi:10.1109/TSG.2015.2427584.

[24] S. Hao, F. Zhuang, New models for integrated short-term forward electricity markets, IEEE Transactions on Power systems 18 (2) (2003) 478-485.

[25] P. B. Luh, Y. Chen, G. A. Stern, W. E. Blankson, F. Zhao, Payment cost minimization with demand bids and partial capacity cost compensations for day-ahead electricity auctions, in: J. Momoh, L. Mili (Eds.), Economic Market Design and Planning for Electric Power Systems, 1st Edition, Wiley, 2009, pp. 71-85. doi:10.1002/9780470529164.ch4.

[26] A.-H. Mohsenian-Rad, V. W. S. Wong, J. Jatskevich, R. Schober, A. Leon-Garcia, Autonomous demand-side management based on game-theoretic energy consumption scheduling for the future smart grid, IEEE Transactions on Smart Grids 1 (3) (2010) 320-331.

[27] J. T. Moore, J. F. Bard, The mixed integer linear bilevel programming problem, Operations Research 38 (5) (1990) 911-921.

[28] J. F. Bard, J. T. Moore, An algorithm for the discrete bilevel programming problem, Naval Research Logistics (NRL) 39 (3) (1992) 419-435.

[29] M. Fischetti, I. Ljubic, M. Monaci, M. Sinnl, Intersection cuts for bilevel optimization, in: IPCO 2016, Liège, Belgium, June 1-3, 2016, Proceedings, 2016, pp. 77-88.

[30] J. V. Outrata, On the numerical solution of a class of stackelberg games, Z. Operations Research 34 (1990) $255-277$.

[31] T. M. Surowiec, Explicit stationarity conditions and solution characterization for equilibrium problems with equilib- 
rium constraints, Ph.D. thesis, Humboldt-Universität zu Berlin (1 2010).

[32] A. Shapiro, On complexity of multistage stochastic programs, Operations Research Letters 34 (2006) 1-8.

[33] R. T. Rockafellar, Solving stochastic programming problems with risk measures by progressive hedging, Set Valued and Variational Analysis (Submitted) (2017) 1-9.

[34] R. Rockafellar, S. Uryas'ev, The fundamental risk quadrangle in risk management, optimization and statistical estimation, Surveys in Operations Research and Management Science 18 (1-2) (2013) 33-53.

[35] R. T. Rockafellar, S. Uryasev, Optimization of conditional value-at-risk, Journal of Risk 2 (2000) $21-41$.

[36] M. Carrión, J. Arroyo, A computationally efficient mixed-integer linear formulation for the thermal unit commitment problem, IEEE Transactions on Power Systems 21 (3) (2006) 1371-1378.

\section{Appendix A. Micro-grids}

For simplicity, this specific study considers the bus model for power flows, that is, the power network is not taken into consideration. We define two types of unit components in the micro-grid, which are called devices in the sequel. Storage devices typically represent batteries, whose status may switch between online (connected to the grid) and offline during the time horizon. During its offline periods, a storage device can be unloaded: for example, an electric vehicle is loaded during the night. During this time period, it can be used to store and serve power, but it must be fully loaded at the end of the night. During the day, the vehicle is used and its battery is emptied so that its storage level is low when it is back online. In our setting, we associate with each storage device a set of online time intervals. At the beginning of such a time interval, the charge level is a stochastic input parameter (for example, it depends on how much the vehicle has been used during the day). The required charge level at the end of the online period is modeled through more general parameters giving minimum and maximum acceptable charge levels for each online time step. We also assume that each storage device has limited capacity, charging and discharging speed and a power loss factor that is the proportion of power stored to the power consumed during the charge.

The regular devices come with stochastic consumptions and productions of power during each time step. Some of their consumption can be partially delayed (elastic demand). We model this feature by defining a set of time intervals for each device (for example, a water heater must heat the water during the night). During each of them, the required total power consumption is known, as a stochastic input data. The maximum power consumption of devices is limited during each time step.

The decisions to be taken in the micro-grid problem are, first, to choose a contract among those proposed by the GenCos. Then, for each regular device and each time step, the amount of elastic power consumption must be determined. For each storage device, the amounts of power consumed (to charge) and released must be fixed. We provide below a model for the problems faced by each one of the micro-grids.

Parameters. Scenario-independent parameters:

- $K$ : set of available contracts, defined by $c_{k}, f_{k}$ and $g_{k}$. We assume that $\forall t \in \mathcal{T}, f_{k t} \geq g_{k t}$.

- $D$ : set of non-storage devices. For each device $d \in D$ :

$-\Theta_{D}^{d}$ : set containing sets of time periods defining elastic consumption slots

- $S$ : set of storage devices. For all $d \in S$ :

$-\bar{s}^{d}$ : capacity of storage device $d$

$-\bar{\ell}^{d}$ : maximum power used to reload $d$ during one time period 
$-\bar{u}^{d}$ : maximum power released by $d$ during one time period

$-\alpha^{d}$ : power loss factor when charging $d$

- $\Theta_{S}^{d}$ : set of time intervals when $d$ is online (can be charged or discharged). We note, for all $\theta \in \Theta_{S}^{d}, \theta=\left[t^{-}(\theta), t^{+}(\theta)\right]$.

$-\underline{\mathrm{S}}_{t}^{d}, \bar{S}_{t}^{d}$ : minimum and maximum charge level for $d$ at time $t$.

Scenario-dependent parameters: For all scenarios $\xi \in \Xi$ :

- For each device $d \in D$ :

$-\forall t \in \mathcal{T}:$

* $b_{t}^{d}(\xi)$ : power production of $d$ during period $t$ in scenario $\xi$

$* r_{t}^{d}(\xi)$ : power consumption of $d$ during period $t$ in scenario $\xi$

$* \bar{w}_{t}^{d}(\xi)$ : maximum possible elastic consumption of $d$ during $t$

$-\forall \theta \in \Theta_{D}^{d}$

$* e_{\theta}^{d}(\xi)$ : total elastic power demand of $d$ during $\theta$ in scenario $\xi$

- For each storage device $d \in S$ and online interval $\theta \in \Theta_{S}^{d}: I_{\theta}^{d}(\xi)$ is the initial stock level when $d$ is plugged in.

Decision variables.

- Stage 0:

- $\forall k \in K: z_{k}=1$ if contract $k$ is chosen by the micro-grid, 0 otherwise

- Stage $t, t \in \mathcal{T}$ :

- $x_{t}(\xi)$ : power consumed by the micro-grid during period $t$

$-y_{t}(\xi)$ : power produced by the micro-grid during period $t$

$* \forall d \in D, w_{t}^{d}(\xi)$ : elastic power consumed by $d$ during $t$

* For all $d \in S$ :

- $s_{t}^{d}(\xi)$ : power stock in $d$ at the end of $t$

- $\ell_{t}^{d}(\xi)$ : power consumed to charge device $d$ during $t$

- $u_{t}^{d}(\xi)$ : power released by discharging device $d$ during $t$ 
Formulation.

$$
\begin{array}{ll}
\min & \mathbb{E}\left[\sum_{k \in K} \sum_{t \in \mathcal{T}}\left(f_{k t} x_{t}(\xi)+g_{k t} y_{t}(\xi)+c_{k}\right) z_{k}\right] \\
\text { s.t. } & \sum_{k \in K} z_{k}=1 \\
& x_{t}(\xi)-y_{t}(\xi)=\sum_{d \in D}\left(r_{t}^{d}(\xi)+w_{t}^{d}(\xi)-b_{t}^{d}(\xi)\right)+\sum_{d \in S}\left(\ell_{t}^{d}(\xi)-u_{t}^{d}(\xi)\right) \quad \forall t, \xi \\
& s_{t}^{d}(\xi)=s_{t-1}^{d}(\xi)+\alpha^{d} \ell_{t}^{d}(\xi)-u_{t}^{d}(\xi) \quad \forall d \in S, \theta \in \Theta_{S}^{d}, t \in \theta-\left\{t^{-}(\theta)\right\}, \xi \\
& s_{t^{-}}^{d}(\theta)(\xi)=I_{\theta}^{d}(\xi)+\alpha^{d} \ell_{t^{-}}^{d}(\theta)(\xi)-u_{t^{-}}^{d}(\theta)(\xi) \quad \forall d \in S, \theta \in \Theta_{S}^{d}, \xi \\
& \sum_{t \in \theta} w_{t}^{d}(\xi)=e_{\theta}^{d}(\xi) \quad \forall d \in D, \theta \in \Theta_{D}^{d}, \xi \\
& w_{t}^{d}(\xi) \leq \bar{w}^{d} \quad \forall d \in D, t, \xi \\
& \underline{S}_{t}^{d} \leq s_{t}^{d}(\xi) \leq \bar{S}_{t}^{d} \quad \forall d \in S, t, \xi \\
& \ell_{t}^{d} \leq \bar{\ell}^{d} \quad \forall d \in S, t \\
& u_{t}^{d} \leq \bar{u}^{d} \quad \forall d \in S, t \\
& \ell_{t}^{d}(\xi)=u_{t}^{d}(\xi)=0 \quad \forall d \in S, \theta \notin \Theta_{S}^{d}, t \in \theta \\
& z_{k} \in\{0,1\} \quad \forall k \in K \\
& x, y, w, r, s, l, u \geq 0
\end{array}
$$

The objective of the problem (Aa) is to minimize the total cost for the micro-grid, which is composed of the fixed cost of the contract, the cost of buying power from the GenCos minus the income obtained from selling the over-production. Constraint (Ab) states that exactly one contract must be chosen. Constraints (Ac) ensure that the power flow into/out of the micro-grid is equal to its production/consumption during each time step. In the right-hand-side, the summation over $D$ (resp. $S$ ) represents the total consumption/production of regular (resp. storage) devices. Constraints (Ad) and (Ae) define the level of power stock for each device and time step. Constraints (Af) fix the correct total amount of power that must be consumed by a device during an elastic consumption interval. The instantaneous power consumed by a device is limited by constraints (Ag). The acceptable stock levels are bound by constraints $(\mathrm{Ah})$. Constraints $(\mathrm{Ai})$ and $(\mathrm{Aj})$ define maximum charging and discharging speeds for the storage devices, while constraints $(\mathrm{Ak})$ are just a way to state that an offline device cannot be charged or discharged (the corresponding variables may as well be omitted in the model). The domains of the variables are given in constraints $(\mathrm{Al})$ and $(\mathrm{Am})$. 